\author{
REVIEW ARTICLE
}

\title{
B-Group vitamin production by lactic acid bacteria - current knowledge and potential applications
}

\author{
J.G. LeBlanc ${ }^{1}$, J.E. Laiño ${ }^{1}$, M. Juarez del Valle ${ }^{1}$, V. Vannini ${ }^{1}$, D. van Sinderen ${ }^{2}$, M.P. Taranto ${ }^{1}$, G. Font \\ de Valdez ${ }^{1,3}$, G. Savoy de Giori ${ }^{1,3}$ and F. Sesma ${ }^{1}$ \\ 1 Centro de Referencia para Lactobacilos (CERELA-CONICET) Chacabuco 145, Tucumán, Argentina \\ 2 Department of Microbiology and Alimentary Pharmabiotic Centre, University College Cork, Cork, Ireland \\ 3 Cátedra de Microbiología Superior, Universidad Nacional de Tucumán (UNT), Tucumán, Argentina
}

\section{Keywords}

biotechnology, dairy, fermented foods,

Lactobacillus, Lactococcus.

\section{Correspondence \\ Jean Guy LeBlanc and Fernando Sesma, CERELA-CONICET, Chacabuco 145, San Miguel de Tucumán, Tucumán T4000ILC, Argentina. \\ E-mails: leblanc@cerela.org.ar, jeanguyleblanc@hotmail.com, fsesma@cerela.org.ar}

2011/1144: received 8 July 2011, revised 31 August 2011 and accepted 11 September 2011

doi:10.1111/j.1365-2672.2011.05157.x

\begin{abstract}
Although most vitamins are present in a variety of foods, human vitamin deficiencies still occur in many countries, mainly because of malnutrition not only as a result of insufficient food intake but also because of unbalanced diets. Even though most lactic acid bacteria (LAB) are auxotrophic for several vitamins, it is now known that certain strains have the capability to synthesize water-soluble vitamins such as those included in the B-group (folates, riboflavin and vitamin $B_{12}$ amongst others). This review article will show the current knowledge of vitamin biosynthesis by LAB and show how the proper selection of starter cultures and probiotic strains could be useful in preventing clinical and subclinical vitamin deficiencies. Here, several examples will be presented where vitamin-producing LAB led to the elaboration of novel fermented foods with increased and bioavailable vitamins. In addition, the use of genetic engineering strategies to increase vitamin production or to create novel vitaminproducing strains will also be discussed. This review will show that the use of vitamin-producing LAB could be a cost-effective alternative to current vitamin fortification programmes and be useful in the elaboration of novel vitaminenriched products.
\end{abstract}

\section{Introduction}

Lactic acid bacteria (LAB) represent a heterogeneous group of micro-organisms that are naturally present in a wide range of ecological niches such as foods and in the gastrointestinal and urogenital tract of animals, including humans. In addition to their important technological properties in food production, several studies have shown that LAB can confer beneficial properties to their hosts, in particular specific members of the genus Lactobacillus, reason for which these bacteria are the most commonly used probiotic micro-organisms. These latter can be defined as 'live microorganisms which when administered in adequate amounts confer a health benefit on the host' (FAO/WHO 2001). Some of the health benefits ascribed to probiotics include promotion of a normal microbiota, prevention of infectious diseases and food allergies, reduction of serum cholesterol, anticarcinogenic activity, stabil- ization of the gut mucosal barrier, immune adjuvant properties, alleviation of intestinal bowel disease symptoms and improvement of the digestion of lactose in intolerant hosts (Ouwehand et al. 2002; Deshpande et al. 2011; Soccol et al. 2011). The probiotic and beneficial aspects of LAB have been extensively reviewed elsewhere and will not be the subject of this review.

Besides probiotic $\mathrm{LAB}$, certain strains of $\mathrm{LAB}$ are able to produce/release and/or increase specific beneficial compounds in foods. These functional ingredients are sometimes referred to as nutraceuticals a term that was first given by Stephen DeFelice in 1989 to describe 'a food (or part of a food) that provides medical or health benefits, including the prevention and/or treatment of a disease.' These ingredients can be macronutrients, micronutrients (such as vitamins) or non-nutritive compounds and can be naturally present in certain foods or added during processing. The proper selection and 
exploitation of nutraceutical-producing micro-organisms is an interesting strategy to produce novel foods with increased nutritional and/or health-promoting properties (Hugenholtz and Smid 2002).

\section{Vitamins}

Vitamins are micronutrients that are essential for the metabolism of all living organisms. They are found as precursors of intracellular coenzymes that are necessary to regulate vital biochemical reactions in the cell. Humans are incapable of synthesizing most vitamins, and they consequently have to be obtained exogenously (i.e. from their diet). Although most vitamins are present in a variety of foods, vitamin deficiencies still exist in many countries including highly industrialized nations mainly because of malnutrition, not only as a result of insufficient food intake but also because of unbalanced diets. The B-group (or B-complex) vitamins include thiamine $\left(B_{1}\right)$, riboflavin $\left(B_{2}\right)$, niacin $\left(B_{3}\right)$, pyridoxine $\left(B_{6}\right)$, pantothenic acid $\left(B_{5}\right)$, biotin $\left(B_{7}\right.$ or $\left.H\right)$, folate $\left(B_{11}-B_{9}\right.$ or $\left.M\right)$ and cobalamin $\left(B_{12}\right)$. Each $B$-group vitamin is chemically different and acts in synergy to maintain the body's homeostasis by playing major roles in metabolic processes such as energy production and red blood cell formation. B-group vitamins, normally present in many foods, are easily removed or destroyed during cooking and food processing, so insufficient intake are common in many societies. For this reason, many countries have adopted laws to enforce the fortification of certain foods with specific vitamins and minerals. For example, in Argentina the food industry is obliged to fortify all wheat flour for human consumption with iron, folic acid, thiamine, riboflavin and niacin to reduce the incidence of anaemia and neural tube deformation. However, recent reports highlighted a lack of official enforcement of the fortification requirement and insufficient fortification levels, and it is therefore not surprising that serum vitamin levels in the general population have only slightly improved and subclinical deficiencies still persist (ENNyS 2007).

Although the beneficial effects of generalized fortification programmes have been demonstrated, such as the decreased incidence of neural tube defects (NTD) and neonatal mortality in countries such as Canada and the USA where folate fortification is mandatory since 1998 (Blencowe et al. 2010), many countries have not adopted a national fortification programme because of possible unwanted side effects. The main concerns are based on the fact that vitamins are added at concentrations that allow persons with low vitamin intakes to reach their recommended daily allowance (RDA) so as to prevent pathologies associated with deficiencies. At these levels of fortification, however, those with normal or elevated lev- els of vitamin ingestion would be subject to excessive intakes. In the case of folic acid fortification, excess intake may in turn mask the early haematological manifestations of vitamin $\mathrm{B}_{12}$ deficiency. This is important as it has been estimated that $10-30 \%$ of people over 50 years have a reduced ability to naturally absorb vitamin $\mathrm{B}_{12}$, and consequently, $20 \%$ of the general population in industrialized countries are potentially deficient for this vitamin (Asrar and O'Connor 2005). As folate fortification levels are based on the requirements of the general population, some groups could be exposed to extremely high levels of folic acid such as children whose vitamin requirements are lower than adults or in pregnant women who take folic acid supplements. It has even been suggested that the foetus may become exposed to excessive amounts of folic acid because of prescribed supplementation of the mother during pregnancy in combination with the consumption of fortified foods, and this could favour the selection of methylentetrahydrofolate polymorphism that is associated with a group of debilitating diseases (Lucock and Yates 2005). As natural folates' such as 5-methyltetrahydrofolate (5-MTHF) that is normally found in foods and sometimes produced by micro-organisms do not mask $B_{12}$ deficiency, this folate form would be a more efficient and secure alternative than supplementation with folic acid (Lamers et al. 2006).

The use of vitamin-producing micro-organisms is thus a more natural and economically viable alternative than fortification with chemically synthesized pseudo-vitamins, and it would allow the production of foods with elevated concentrations of vitamins that are less likely to cause undesirable side effects.

\section{Riboflavin}

Riboflavin (vitamin $\mathrm{B}_{2}$ ) plays an essential role in cellular metabolism, being the precursor of the coenzymes flavin mononucleotide (FMN) and flavin adenine dinucleotide (FAD) both acting as hydrogen carriers in biological redox reactions involving enzymes such as nicotinamide adenine dinucleotide (NADH) dehydrogenase. Here, the term 'riboflavin' is used to describe all biologically active forms of vitamin $\mathrm{B}_{2}$ flavins, including riboflavin (7,8-dimethyl10 -(1'-D-ribityl) isoalloxazine), riboflavin- $5^{\prime}$-phosphate $($ FMN) and riboflavin-5'-adenosyldiphosphate (FAD). The recommended riboflavin requirements for humans vary with respect to sex, ageand physiological state (pregnancy and lactation). Normal adults need to consume between 0.9 and $1.6 \mathrm{mg}$ of this vitamin on a daily basis as the human body cannot adequately store riboflavin (Institute of Medicine 1998). Although present in a wide variety of foods, such as dairy products, meat, eggs and certain green vegetables, riboflavin deficiency (ariboflavinosis) still 
occurs in both developing and industrialized countries (O'Brien et al. 2001; Blanck et al. 2002). Symptoms of ariboflavinosis in humans include sore throat, hyperaemia, oedema of oral and mucous membranes, cheilosis and glossitis (Wilson 1983). Severe cases of ariboflavinosis are not common in most societies; however, subclinical manifestations are frequent amongst all subpopulation groups. Subclinical riboflavin deficiencies are only detectable by measuring the vitamin concentration in body fluids such as blood plasma and serum. Vitamin $B_{2}$ status in humans has usually been assessed by measuring the erythrocyte glutathione reductase activation coefficient (EGRAC), which is the ratio between glutathione reductase activity determined with and without the addition of the cofactor, FAD (Glatzle et al. 1970). Glutathione reductase loses FAD at an early stage in vitamin $B_{2}$ deficiency, making EGRAC a useful method for the diagnosis of vitamin $B_{2}$ deficiency (Bates 1993).

Although dairy products contain riboflavin, they are not considered a good source of this essential vitamin. Considering that milk contains $c .1 \cdot 2 \mathrm{mg}$ of riboflavin per litre, an average adult person and a pregnant woman would need to consume, respectively, 1 and 1.61 of milk per day to meet their daily requirement. This level of milk consumption far exceeds that of residents of industrialized countries such as USA where the daily per capita consumption of fresh milk is $c$. $200 \mathrm{ml}$ (Putman and Allshouse 2003). Increasing the levels of riboflavin in milk would thus be very important to prevent ariboflavinosis in populations where milk consumption is low.

Riboflavin biosynthesis has been described both in grampositive and gram-negative bacteria, with detailed studies performed for Bacillus subtilis (Perkins and Pero 2002) and Escherichia coli (Bacher et al. 1996). Microbial biosynthesis of riboflavin from the precursors guanosine triphosphate (GTP) and D-ribulose 5-phosphate occurs through seven enzymatic steps that have previously been reviewed in detail (Bacher et al. 2000). The imidazole ring of GTP is hydrolytically opened, yielding a 4,5-diaminopyrimidine, which is converted to 5-amino-6-ribitylamino-2,4 $(1 \mathrm{H}, 3 \mathrm{H})$ pyrimidinedione by a sequence of deamination, sidechain reduction and dephosphorylation. Condensation of 5-amino-6-ribitylamino-2,4 $(1 \mathrm{H}, 3 \mathrm{H})$-pyrimidinedione with 3,4-dihydroxy-2-butanone 4-phosphate obtained from ribulose 5-phosphate affords 6,7-dimethyl-8-ribityllumazine. Dismutation of the lumazine derivative yields riboflavin and 5-amino-6-ribitylamino-2,4 $(1 \mathrm{H}, 3 \mathrm{H})$-pyrimidinedione, which is recycled in the biosynthetic pathway.

Riboflavin concentrations can sometimes vary in certain dairy products (see Fig. 1) because of processing technologies and through the action of micro-organisms utilized during food processing(LeBlanc et al. 2010b). This is the case for buttermilk and yogurt where ribofla-

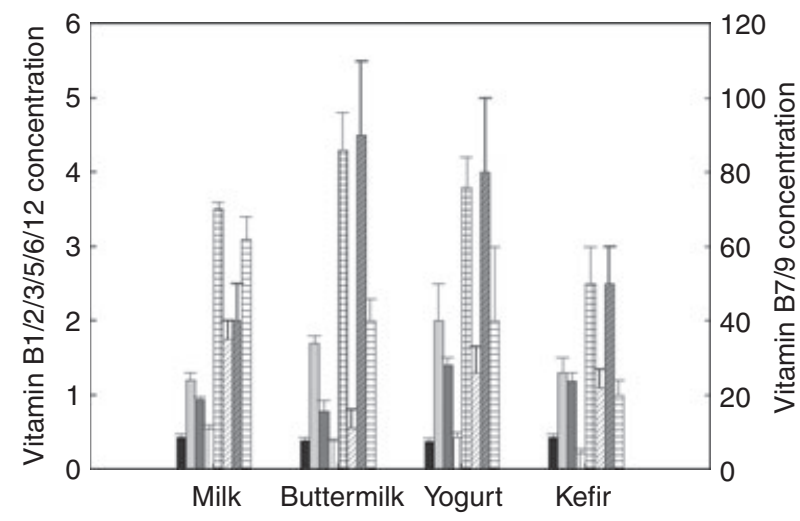

Figure 1 Vitamin concentrations in dairy products (modified from Alm (1982) and LeBlanc et al. 2010b). Concentrations of thiamine $\left(B_{1}, \square\right)$, riboflavin $\left(B_{2}, \square\right)$, niacin $\left(B_{3}, \square\right)$, pyridoxine $\left(B_{6}, \square\right)$ and pantothenic acid $\left(B_{5}, \mathbb{N}\right)$ are in $\mathrm{mg} \mathrm{l}^{-1}$, whereas biotin $\left(B_{7}, \square\right)$, folate $\left(\mathrm{B}_{9} / \mathrm{B}_{11}, \mathbb{Z}\right)$ and cobalamin $\left(\mathrm{B}_{12}\right.$, 目) are in $\mu \mathrm{g} \mathrm{I}^{-1}$.

vin levels increased significantly $\left(1 \cdot 7\right.$ and $\left.2 \cdot 0 \mathrm{mg}^{-1}\right)$ compared with unfermented milk $\left(1 \cdot 2 \mathrm{mg} \mathrm{l}^{-1}\right)$. It has been shown that most yogurt starter cultures decreased riboflavin concentrations whereas others can increase the levels of this essential vitamin up to $60 \%$ of the initial concentration present in unfermented milk (Kneifel et al. 1992).

In a clinical trial, it was shown that daily consumption of $200 \mathrm{~g}$ of both a probiotic or conventional yoghurt for 2 weeks can contribute to the total intake of vitamin $B_{2}$, as reflected by increased levels of plasma-free riboflavin in healthy women (Fabian et al. 2008). However, as riboflavin levels returned to normal when the intake of fermented milks was stopped (no long-term effect), the changes in plasma concentrations seem more likely the result of regular yoghurt consumption as a fermented dairy product, rather than of the specific intake of the probiotic bacteria (Fabian et al. 2008). Previous results from this group showed that unlike some yoghurt starter cultures that are able to produce riboflavin, most probiotic strains of lactobacilli consume this vitamin and thus decrease their bioavailability in fermented products (Elmadfa et al. 2001). Consequently, adequate selection of strains is essential to increase the concentration and bioavailability of this essential vitamin in fermented foods.

Tempeh, a traditional Indonesian fermented soybean food, was shown to contain elevated concentrations of Bgroup vitamins (such as riboflavin) because of microbial biosynthesis (Keuth and Bisping 1993). The latter article also reported on the isolation of LAB from tempeh, which were shown to belong to the Streptococcus and Enterococcus genera and able to significantly increase riboflavin concentrations in this fermented product. 
Recently, a publication has described the screening of riboflavin-producing strains from different fermented milk products obtained in the Vellore region of India (Jayashree et al. 2010). Half of the 48 isolates were able to grow in a chemically defined medium (CDM) without riboflavin although just a single strain was identified as being an efficient riboflavin-producing strain: Lactobacillus fermentum MTCC 8711 produced $2 \cdot 29 \mathrm{mg} \mathrm{l}^{-1}$ of riboflavin after $24 \mathrm{~h}$ of growth in the CDM (Jayashree et al. 2010). These authors conclude that this strain could be further exploited for the enhanced production of riboflavin using various strain improvement strategies to develop a better starter culture for the fermented food industry. They also propose that it could be used to replace the conventional strains that are being employed in these LAB-based fermented products.

The selection of spontaneous roseoflavin-resistant mutants was found to be a reliable method to obtain natural riboflavin-overproducing strains of a number of species commonly used in the food industry (Burgess et al. 2006). The toxic riboflavin analogue roseoflavin was used to isolate natural riboflavin-overproducing variants of the food-grade micro-organisms Lactococcus lactis (Burgess et al. 2004), Lactobacillus plantarum, Leuconosctoc mesenteroides and Propionibacterium freudenreichii (Burgess et al. 2006). In these studies, it was demonstrated that spontaneous resistance to the toxic riboflavin analogue roseoflavin frequently coincides with a riboflavin-overproducing phenotype because of mutations in the regulatory region of the rib operon.

Recently, LAB were obtained from durum wheat flour samples and screened for roseoflavin-resistant variants to isolate natural riboflavin-overproducing strains (Capozzi et al. 2011). Two riboflavin-overproducing strains of Lact. plantarum were isolated and used for the preparation of bread (by means of sourdough fermentation) and pasta (using a prefermentation step) to enhance their vitamin $\mathrm{B}_{2}$ content. The applied approaches resulted in a considerable increase in vitamin $\mathrm{B}_{2}$ content (about a two and threefold increase in pasta and bread, respectively), thus representing a convenient and efficient food-grade biotechnological application for the production of vitamin $\mathrm{B}_{2}$-enriched bread and pasta.

The roseoflavin-resistant, riboflavin-producing strain L. lactis CB010 was able to eliminate most physiological manifestations of ariboflavinosis such as stunted growth, elevated EGRAC values and hepatomegalia that were observed using a riboflavin depletion-repletion animal model (LeBlanc et al. 2005a). The bioavailability of the riboflavin produced by this strain was similar to that of pure riboflavin demonstrating the usefulness of this strain for the development of riboflavin-enriched fermented foods.
In another study, it was shown that the administration of a fermented milk that was produced with the roseoflavin-resistant and spontaneous riboflavin-overproducing strain $P$. freudenreichii B2336 was beneficial to riboflavindepleted animals (LeBlanc et al. 2006). The fermented product containing $P$. freudenreichii B2336, with increased concentrations of riboflavin, eliminated most physiological manifestations of ariboflavinosis, whereas a product fermented with a non-riboflavin-producing strain of $P$. freudenreichii did not show this beneficial effect (LeBlanc et al. 2006).

Another method to obtain riboflavin-producing strains or to increase their production capacities is to use metabolic engineering strategies. A Corynebacterium ammoniagenes strain harbouring a plasmid containing all of its riboflavin biosynthetic genes was constructed through metabolic engineering using recombinant DNA techniques. This recombinant strain was shown to produce and accumulate riboflavin to levels that were 17-fold higher as compared to the plasmid-free parent strain (Koizumi et al. 2000).

By means of classical mutagenesis and gene technology, the gram-positive bacterium B. subtilis was modified to become a suitable host for the commercial production of riboflavin (Perkins et al. 1999). A sequential optimization strategy, based on statistical experimental designs, was used to enhance the production of riboflavin by recombinant B. subtilis RH44 (Wu et al. 2007). Recently, enhanced riboflavin production was obtained by expressing heterologous riboflavin operon from Bacillus cereus ATCC14579 in B. subtilis (Yunxia et al. 2010).

Previously, we described the genetic analysis of the riboflavin biosynthetic ( $r i b$ ) operon in the lactic acid bacterium L. lactis ssp. cremoris strain NZ9000 (Burgess et al. 2004). This strain was converted from a riboflavin consumer into a vitamin $\mathrm{B}_{2}$ 'factory' by overexpressing its riboflavin biosynthesis genes (Burgess et al. 2004). Substantial riboflavin overproduction $\left(24 \mathrm{mg} \mathrm{l}^{-1}\right)$ was described when all four biosynthetic genes ( $r i b G$, ribH, ribB and ribA) were overexpressed simultaneously (in L. lactis NZ9000 containing pNZGBAH). It was demonstrated that milk fermented by this genetically modified riboflavin-producing strain was effective in reversing ariboflavinosis in a riboflavin-deficiency rat model (LeBlanc et al. 2005b). The manufacture of a product of this nature would decrease the costs compared with current vitamin fortification programmes.

A great advantage of genetic engineering strategies is that more than one functional property can be conferred to the host micro-organisms. By directed mutagenesis followed by selection and metabolic engineering, folate and riboflavin biosynthetic pathways were modified in L. lactis resulting in simultaneous overproduction of both folate and riboflavin (Sybesma et al. 2004). According to these 
authors, novel foods, enriched through fermentation using these multivitamin-producing starters, could compensate the B-vitamin-deficiencies that are common even in highly developed countries.

\section{Folates}

Because folate is involved in essential functions of cell metabolism such as DNA replication, repair and methylation and synthesis of nucleotides, vitamins and some amino acids, human life could not exist without it. Folate deficiency has been implicated in a wide variety of disorders from Alzheimer's to coronary heart diseases: osteoporosis, increased risk of breast and colorectal cancer, poor cognitive performance, hearing loss and NTDs (LeBlanc et al. 2007, 2010b; Laiño et al. 2011). In this review, the generic term folate will include the complete group of all natural folate derivatives, including 5-methyltetrahydrofolate (5-MTHF) and folylglutamates that are naturally present in foods, but not folic acid, that is the synthetic form of folate commonly used for food fortification and nutritional supplements.

Considering that milk contains between 20 and $50 \mu \mathrm{g} \mathrm{l}^{-1}$ of folate, an average adult person or a pregnant woman would need to consume 6-12 1 of milk per day to meet their daily requirement, and as this level of consumption is unrealistic, it may be helpful to increase folate intake using vitamin-producing micro-organisms to prevent the occurrence of folate deficiency.

Many industrially important LAB such as L. lactis and Streptococcus thermophilus have the ability to synthesize folate (Friend et al. 1983; Lin and Young 2000; Hugenholtz and Smid 2002; Crittenden et al. 2003; Sybesma et al. 2003c; Papastoyiannidis et al. 2006). This explains why some fermented dairy products, including yogurt, contain higher amounts of folate compared with nonfermented milks (see Fig. 1). It was shown that folate concentration in yogurt may be increased to values above $200 \mu \mathrm{g} \mathrm{l}^{-1}$ (Wouters et al. 2002). However, the ability of microbial cultures to produce or utilize folate varies considerably being a strain-dependent trait. Most authors claim that Strep. thermophilus normally produce folates whereas Lactobacillus delbrueckii subsp. bulgaricus is a folate consumer, so the selection of adequate combination of strains is essential to develop fermented foods with increased vitamin concentrations.

Not only Strep. thermophilus and L. lactis have the ability to produce folates, but also other LAB like Lactobacillus acidophilus and Lact. plantarum have been reported to produce folate in CDM (LeBlanc et al. 2010b) as have Leuconostoc lactis and Bifidobacterium longum. Also, Lactobacillus reuteri JCM1112, a well-known producer of vitamin $B_{12}$, can produce high quantities of folates, so this
LAB could potentially increase folate levels in milk (Santos et al. 2008b).

Another example of LAB producing folates is the combination of Strep. thermophilus and Bifidobacterium animalis that increased the levels of this vitamin sixfold (Crittenden et al. 2003). It is well established that Strep. thermophilus strains are dominant producers of folates in milk, principally producing 5-MTHF, what leads to yogurts with more than six times the 5-MTHF content as compared to the control after $12 \mathrm{~h}$ of fermentation (Holasova et al. 2004). Also it was found that some strains of $B$. longum were moderate producers with a maximum increase of $73 \%$ in 5-MTHF after this fermentation time. On the other hand, Propionibacterium freundenreichii ssp. shermanii strains did not modify folate levels. The maximum concentration of 5-MTHF was highest between 6 and $12 \mathrm{~h}$ of fermentation, then a decrease was observed (Holasova et al. 2004).

Micro-organisms are also able to increase folate content in a wide variety of other foods. For example, fermentation of rye dough to produce bread is frequently accompanied by increases in folate concentrations (Kariluoto et al. 2006), but the increase in this vitamin during fermentation was shown to be mainly because of folate synthesis by yeasts, whereas LAB did not produce folate, they consumed it. So replacing folate consumers for folate-producing LAB could significantly increase folate content in these breads.

It has also been reported that it is possible to select starter cultures of LAB that produce significant amounts of 5-MTHF (to almost twice the basal level) during vegetable fermentation (Jägerstad et al. 2004). It is important to carefully check the folate concentration in raw vegetables to optimize the entire process. Folate losses during processing must be limited as much as possible, and optimizing the conditions to favour the microbiological biosynthesis of folates is essential to increase folate levels in the final product.

Another example of the use of LAB to improve folate content in fermented products is in the fermentation of corn flour where an increase in folate level of almost threefold after 4 days of fermentation at $30^{\circ} \mathrm{C}$ was achieved (Murdock and Fields 1984).

Some studies performed with the aim to determine whether the exogenous vitamin can affect folate synthesis by bacteria have shown that production is strain dependent; some bifidobacteria did not produce folate when this vitamin was already present, whereas others produced it regardless of the vitamin concentration. This suggests that in some strains folate biosynthesis might not be regulated; this was confirmed by the finding that the final concentration of this vitamin was at least 50-fold higher than the requirement after bacterial growth of the folateproducing strains (Pompei et al. 2007). 
Different forms of folates are produced by LAB; some even produce folates with more than three glutamyl residues. In L. lactis, up to $90 \%$ of the total produced folate remains in the cell as 5,10-methenyl-THF and presumably 10-formyl-THF, both with four, five or six glutamate residues (Sybesma et al. 2003c). In Strep. thermophilus, much less was shown to remain in the cell, with this folate being present as 5-formyl-THF and 5,10-methenyl-THF, both with three glutamate residues. The differences in distribution can probably be explained by the different length of the polyglutamyl tail in the two micro-organisms. One of the main functions of the polyglutamyl tail is thought to be the retention of folate within the cell. The cell retention of folate can be a result of the negative charge of the carboxyl groups of (polyglutamyl) folate (pKa of 4.6). Moreover, in Strep. thermophilus, the intraand extracellular folate distribution was influenced by the $\mathrm{pH}$. Cells that grew at low $\mathrm{pH}$ had a larger extracellular folate fraction than cells that were cultured at high $\mathrm{pH}$. The explanation can be that at low intracellular $\mathrm{pH}$, folate is protonated and so became electrically neutral, enhancing transport across the membrane. In the case of L. lactis, $\mathrm{pH}$ did not seem to affect intra- and extracellular folate distribution (Sybesma et al. 2003c).

The genes for folate biosynthesis have been identified in L. lactis (Sybesma et al. 2003a), in Lact. plantarum (Kleerebezem et al. 2003) and in Lact. delbrueckii ssp. bulgaricus (van de Guchte et al. 2006), but in the last one, some of them are missing. Not every Lactobacillus is able to produce folate because the genes involved in folate biosynthesis are lacking in the genome; this is the case for Lactobacillus gasseri (Wegkamp et al. 2004), Lactobacillus salivarius (Claesson et al. 2006), Lact. acidophilus and Lactobacillus johnsonii (van de Guchte et al. 2006).

In cells, the polyglutamyl form is the main form as folate-dependent enzymes have increased affinity for polyglutamyl folates compared with the monoglutamyl forms. The enzyme responsible for polyglutamyl folate synthesis and the corresponding elongation of the chain is polyglutamyl synthetase, encoded by the folC gen in L. lactis. All sequenced microbial genomes (even those of strains not able to produce folate) possess folC or a homologous gene (Sybesma et al. 2003b).

Through metabolic engineering, it is possible to increase folate levels in L. lactis (Sybesma et al. 2003b; Wegkamp et al. 2007), Lact. gasseri (Wegkamp et al. 2004) and Lact. reuteri (Santos et al. 2008b). By controlling the overexpression of folKE genes in L. lactis that encode 6-hydroxymethil-dihydropterinpyrophosphokinase $(f \circ l K)$ and GTP cyclohydrolase $(f \circ l E)$ results in a tenfold increased production of extracellular folate and a threefold increased production of total folates; meanwhile, overexpression of folA that encode dihydrofolate reduc- tase decreased (by 50\%) the production of total folates. In addition, it was observed that the combined overexpression of folKE and folC favoured the accumulation of intracellular folate (Sybesma et al. 2003b). Furthermore, the overexpression of GTP cyclohydrolase I showed a very promising potential to increase the flux through the folate biosynthesis pathway. In consequence, the appropriate combination of the overexpression of folKE with the increased or decreased expression of other folate biosynthesis genes can significantly increase folate production (Sybesma et al. 2003b).

An example of that is the significantly improved folate status in deficient rats that was shown upon supplementation with L. lactis overexpressing the folC, folKE or folC + folKE genes (LeBlanc et al. 2010a). The biosafety assessment of these genetically modified LAB (GM-LAB) was performed and demonstrated that there were as safe as the native strains from which they were derived (LeBlanc et al. 2010c).

Increases in folate production can be performed not only by overexpressing the genes involved in the biosynthesis, but also by overexpressing other genes involved in the biosynthesis pathway of related metabolites. For example, the overproduction of pABA did not lead to elevated folate pools on its own (Wegkamp et al. 2007). However, simultaneous overexpression of the pABA and the folate biosynthesis gene clusters reached high folate levels (Wegkamp et al. 2007), which did not depend of pABA supplementation. The overproduction of $p A B A$ led to relatively low intracellular folate pools and a relatively high secretion of folate. There exists a very tight correlation between folate and pABA biosynthesis that was shown through deletion of the pABA genes in L. lactis where in consequence its ability to synthesize folate was eliminated, causing a complete inability to grow in the absence of purine nucleobases/nucleosides.

In other trials, Lact. gasseri ATCC 33323 was converted from being a folate consumer into a highly efficient folate-producing strain (Wegkamp et al. 2004). In this strain, the folate biosynthesis genes are not present, except for folA and folC, which are involved in the regeneration and retention of reduced folates absorbed from the medium. When a plasmid containing the complete folate gene cluster ( $f \circ l A, f o l B$, folKE, folP, ylgG and folC) from L. lactis MG1363 was introduced into Lact. gasseri ATCC 33323, the resulting recombinant strain was converted into a folate-producing bacterium (Wegkamp et al. 2004).

Although it is useful during technological applications to increase folate production, a recent study has been shown that folate overproduction in Lact. plantarum WCFS1 significantly reduced the growth rate of this micro-organism (Wegkamp et al. 2010). Even when folate 
overproduction led to very little change in metabolite levels or overall transcript profile, the growth rate in Lact. plantarum was reduced drastically, most likely because the growth-related transcripts and proteins are diluted by the enormous amount of gratuitously produced folate-related transcripts and proteins (Wegkamp et al. 2010). The results obtained in this study will be helpful in designing future genetic engineering strategies taking into account that transcript numbers can affect growth.

In animal studies, it was shown that low-folate diets are associated with an elevated risk of colorectal cancer and that folic acid would suppress the growth of the cancer (Giovannucci 2002). The use of folate-producing probiotics have recently been proposed to efficiently confer protection against inflammation and cancer, both exerting the beneficial effects of probiotics and preventing the folate deficiency that is associated with premalignant changes in the colonic epithelia (Rossi et al. 2011). However, large discrepancies exist between folate supplementation and cancer prevention: some researchers have demonstrated a clear reduction in the risk of recurrence of adenomas with increased folate intakes, whereas others observe the complete opposite (Carroll et al. 2010). It is all these conflicting results that have raised concerns the implementation of new fortification policies of many countries (Ulrich 2008). It is thus very important to establish the risk-benefit relationship of folate and folic acid supplementation before proposing their use as a chemopreventive agent.

\section{Vitamin $\mathbf{B}_{12}$}

The term vitamin $B_{12}$ is generally used to describe a type of cobalt corrinoid, particularly of the cobalamin (cbl) group. In strict terms, vitamin $\mathrm{B}_{12}$ is the form of the vitamin obtained during industrial production and which does not exist naturally (Rucker et al. 2001). Cyanide stabilizes the molecule during the extraction procedure from microbial cultures, forming cyanocobalamin. In its natural form, the vitamin is present principally as desoxyadenosilcobalamin (coenzyme $\mathrm{B}_{12}$ ), methylcobalamin or pseudocobalamin, amongst other forms. Animals, plants and fungi are incapable of producing cobalamin; it is the only vitamin that is exclusively produced by micro-organisms, particularly by anaerobes (Roth et al. 1996; Martens et al. 2002; Smith et al. 2007). Furthermore, biochemical and genomic data indicate that only a few bacteria and archaea possess the ability to produce this vitamin (Roth et al. 1996; Rodionov et al. 2003). Adult ruminant animals and strict vegetarians can obtain the vitamin in specialized bacteria present in the rumen. Humans, however, do not harbour such microbes in their small intestine and must absorb the coenzyme from natural sources such as animal meats (especially liver and kidney), fish and eggs, or pharmaceutical products. Vitamin $\mathrm{B}_{12}$ deficiency can cause different pathological manifestations that affect the haematopoietic, neurological and cardiovascular system, amongst others. One of the most extreme forms of $\mathrm{B}_{12}$ deficiency is known as pernicious anaemia that is not normally associated with diet but rather with problems in the gastric system caused by a lack of production of a gastric glycoprotein called intrinsic factor that facilitates the absorption of the vitamin in the small intestine (Beck 2001).

As indicated previously, only bacteria and archaea are able to synthesize vitamin $\mathrm{B}_{12}$, although relatively few can synthesize it de novo. One of the first model organisms used for the study of $\mathrm{B}_{12}$ biosynthesis was $P$. freudenreichii that is used in the industrial production of the vitamin. To circumvent the instability of the biosynthetic intermediates, the aerobic $\mathrm{B}_{12}$-producing bacterium Pseudomonas denitrificans has been used for the isolation of various intermediates and the characterization of the majority of the genes and corresponding products involved in the biosynthesis of this vitamin (Battersby 1994; Thibaut et al. 1998).These studies concluded that the biosynthesis of cobalamin could be performed under either aerobic (oxygen dependent) or anaerobic (oxygen independent) conditions.

The anaerobic route was observed in the strains of P. freudenreicchii, Salmonella enterica and Bacillus megaterium (Warren et al. 2002; Warren 2006; Escalante-Semerena 2007). The initial characterization problems were primarily due to the fact that the central $\mathrm{Co}^{2+}$ ion was inserted into the corrinoid ring in an early step that generated unstable intermediates that were difficult to isolate. On the other hand, in the oxygen-dependent route, cobalt is inserted in a later stage creating more stable intermediates.

Because of the complexity of $\mathrm{B}_{12}$ biosynthesis and the limitations of the scope of this chapter, interested readers are invited to read excellent reviews that have been published on this subject (Raux et al. 2000; Scott 2003; Roessner and Scott 2006; Escalante-Semerena 2007).

It was shown that Lact. reuteri CRL1098 was able to metabolize glycerol in a $\mathrm{B}_{12}$-free medium; this being the first hint that a LAB might be able to produce cobalamin (Taranto et al. 2003). The chromatographic analysis of the intracellular bacterial extract of Lact. reuteri CRL 1098 confirmed that this strain was able to produce a cobalamin-like compound with an absorption spectrum closely resembling that of standard cobalamin but with a different elution time, while cobalamin production was confirmed using different bioassays (Taranto et al. 2003).

Genetic evidence of cobalamin biosynthesis by Lact. reuteri CRL 1098 was then obtained through the use of different molecular biology techniques, and it was shown that at least 30 genes are involved in the de novo 
synthesis of the vitamin. The genetic organization ( $c o b$ and $c b i$ genes) are very similar to those of Salm. enterica and Listeria innocua (Santos et al. 2007).

One distinctive characteristic of the cob cluster of Lact. reuteri is the presence of hem genes in the middle of the cluster. In the respiratory organisms Listeria and Salmonella with similar cob clusters, the hem genes are located at other positions of their genome. The presence of the hem genes in the cob cluster is a characteristic that has only been observed in certain genomes of Clostridium (Rodionov et al. 2003). Recently, the transcription of a vast set of genes involved in cobalamin synthesis in sourdough prepared with strain Lact. reuteri ATCC 55730 was described (Hufner et al. 2008).

In addition to CRL1098, other Lact. reuteri strains were shown to be capable of producing some corrinoids such as Lact. reuteri DCM 20016 (Santos et al. 2008a), JCM1112 (Santos et al. 2008b) and CRL 1324 and 1327, strains isolated from human vagina (Vannini et al. 2008). After the detection of $\mathrm{B}_{12}$ production by Lact. reuteri CRL1098 and the study of its cobalamin biosynthesis cluster, the genomic sequence of two strains of Lact. reuteri, with different characteristics, was released by the Joint Genome Institute: Lact. reuteri F275 (type stain DSM20016) isolated from human faeces that is unable to colonize mice and Lact. reuteri 100-23 isolated from the mouse intestine. Curiously, comparative genomic data revealed that the strain isolated from the human intestine (DSM 20016) contains the cobalamin biosynthesis cluster, which is associated with the anaerobic catabolism of glycerol (or 1,2propanediol), whereas the mouse strain (100-23) neither contained the $c o b$ nor the glycerol metabolism genes. Based on the horizontal transfer hypothesis of the cob-pdu cluster, it would be expected that other strains of LAB would also have received this genomic island by one of the many mechanisms of genetic transfer. Notably, of the current sequenced genomes of LAB, only Lact. reuteri contain the pdu-cob genes (DSM20016/JCM1112 and the Biogaia strain Lact. reuteri ATCC55730). Recently, a reuterinproducing strain of Lactobacillus coryniformis isolated from goat milk was characterized and was shown to produce a cobalamin-type compound (Martin et al. 2005). Preliminary genetic and biochemical data from our laboratory (Vannini et al. 2008) indicate that the cob-pdu cluster is indeed present in other lactobacilli (Lact. coryniformis and Lactobacillus murinus). The possibility of various vitamin $B_{12}$-producing strains and species of LAB is important for future studies on cobalamin production, not only in evolutionary studies to address how the cob-pdu genomic island was acquired, but also to explore its potential application in the development of products that contain $\mathrm{B}_{12}$.

Recently, a vitamin $B_{12}$-deficient murine experimental model was developed to evaluate maternal $\mathrm{B}_{12}$ deficiency from the end of the gestation period to weaning (Molina et al. 2008). In this experimental animal model, vitamin $\mathrm{B}_{12}$ deficiency caused a significant reduction in the haematological parameters (haemoglobin, haematocrit and reticulocytes values) and anthropometric alterations in pregnant females compared with the control animals, which were fed a $\mathrm{B}_{12}$-sufficient diet. Moreover, the deficient females gave birth to smaller numbers of offspring, which also showed growth retardation (smaller size) and a decrease in haematological values with associated histological alterations in the small intestine and a decrease in the number of IgA-producing cells of the females and in their offspring. This experimental model of murine females and their offspring allowed the assessment of the incidence of maternal cobalamin deficiency in offspring, probably also representing a useful tool to evaluate the efficiency of functional foods containing $\mathrm{B}_{12}$-producing micro-organisms to prevent the nutritional deficit of cobalamin. Using this model it was shown that Lact. reuteri CRL 1098 was able to revert vitamin $B_{12}$ deficiency, demonstrating the bioavailability of the vitamin produced by this strain (Molina et al. 2009). It is known that when Lact. reuteri CRL 1098 is grown in strict anaerobiosis, this micro-organism produces almost exclusively pseudocobalamin (Santos et al. 2007), a variant of the vitamin that appears to be inactive in animals, though, relevant in the microbial studies. Salmonella enterica serovar typhimurium, a $\mathrm{B}_{12}$-producing facultative anerobic bacterium, synthesizes pseudocobalamin in anaerobiosis, but in microaerophilic conditions the coenzyme $\mathrm{B}_{12}$ is also produced (Keck et al. 1998). Preliminary studies from our laboratory indicate that an additional corrinoid compound (with an absorption spectrum similar to cobalamin) is produced when Lact. reuteri is grown in microaerophilic conditions (V. Vannini, G. Font de Valdez, P. Taranto and F. Sesma, unpublished data). These results may explain the results from the biodisponibility assays and emphasize the need for further metabolic studies to establish better conditions for the production of the active form of the vitamin.

A common method to improve $B_{12}$ yields is random mutagenesis and the use of genetic engineering (Martens et al. 2002; Burgess et al. 2009). Different metabolic engineering strategies have been applied to increase vitamin $\mathrm{B}_{12}$ production in $P$. freudenreichii (Piao et al. 2004a,b). A recombinant $P$. freudenreichii strain harbouring a plasmid containing hemA, from Rhodobacter sphaeroides, and homologues of hemB and cobA showed 2-2-fold overproduction of vitamin $B_{12}$ (Piao et al. 2004b). These studies show that multigene expression systems improve the vitamin $B_{12}$ production levels in propionibacteria. On the other hand, no similar studies have been conducted in lactobacilli. Knowledge of multiple genomic sequences of 
Lact. reuteri $\mathrm{B}_{12}$-producing strain will facilitate the design of strategies for constructing food-grade strains with enhanced capacity to produce this essential vitamin.

\section{Other B-group vitamins}

Besides riboflavin, folate and vitamin $\mathrm{B}_{12}$, increased levels of other B-group vitamins such as niacin and pyridoxine have also been reported as result of the LAB fermentation in yoghurt, cheeses and other fermented products (Shahani and Chandan 1979; Alm 1982). The concentration of thiamine in milk was also positively influenced $(11 \%$ increase) following $48 \mathrm{~h}$ of fermentation with B. longum (Hou et al. 2000). Recently, it was shown that a slight (but not statistically significant) increase in the thiamine and pyridoxine concentration occurred as a result of soy fermentation with Strep. thermophilus ST5 and Lactobacillus helveticus R0052 or B. longum R0175 (Champagne et al. 2010). These authors state that their strains were not as efficient, as those reported in literature, but clarify that in these previous studies, fermentations had been carried out in milk-based products, and results were not subjected to statistical analyses. The authors then conclude that this was the first study on thiamine and pyridoxine amounts in soy beverages fermented by pure cultures of Lact. helveticus and Strep. thermophilus.

\section{Other vitamins}

Although B-group vitamins are the most commonly studied because of their importance in nutrition and general metabolism, a few reports have shown that other watersoluble vitamins can be produced by LAB. In one such study, LAB were examined for their ability to produce quinone compounds as vitamin $\mathrm{K}$ occurs naturally in two forms, namely, K1 (phylloquinone) in green plants and K2 (menaquinones) in animals and some bacteria (Morishita et al. 1999). Lactococcus lactis ssp cremoris (three strains), L. lactis ssp lactis (two strains) and Leuc. lactis were selected as high producers of quinone that synthesized more than $230 \mathrm{nmol}$ of quinones $\mathrm{g}^{-1}$ of dried cells. These strains, when grown either in reconstituted nonfat dry milk or in a soymilk medium, produced a beneficial quantity for dietary supplement (i.e. 29-123 $\mu$ g of menaquinones $\mathrm{l}^{-1}$ of the fermented medium) (Morishita et al. 1999). Vitamin $K$ is an essential cofactor for the formation of $\gamma$-carboxyglutamic acid (Gla) residues in proteins, and these can bind calcium ions and influence, for example, blood coagulation and tissue calcification (e.g. osteocalcin found in bone tissues). Vitamin $\mathrm{K}$ deficiency has been implicated in several clinical ailments such as intracranial haemorrhage in newborn infants and possible bone fracture resulting from osteoporosis. The menaqui- none-producing LAB strains identified by Morishita et al. could thus be useful to supplement vitamin $\mathrm{K}$ requirement for humans.

\section{Conclusions}

This review describes the possibility to increase vitamin concentrations in fermented foods through judicious selection of the microbial species and cultivation conditions. It is expected that the food industry will take the next step to use this information for selecting vitaminproducing strains as part of their starter cultures to produce fermented products with elevated levels of these essential compounds. Such products would provide economic benefits to food manufacturers as increased 'natural' vitamin concentrations would be an important value-added effect without increasing production costs. Consumers would obviously benefit from such products as they could increase their vitamin intakes while consuming them as part of their normal diet.

With the increased availability of genome sequences and the development of novel engineering tools for LAB, the manufacture of products using GM vitamin-producing strains is now possible, and these are a viable costeffective alternative to current vitamin fortification programmes. The ultimate use of such GM-LAB may rely on the regulatory acceptance of genetically modified organisms in nutrition and nutraceutical preparations. Undoubtedly, consumers will play a decisive role in such a decision, and their position should be guided by the safety status of such genetically modified organisms and their associated, scientifically proven health benefits.

\section{Acknowledgements}

The authors would like to thank the Consejo Nacional de Investigaciones Científicas y Técnicas (CONICET), Agencia Nacional de Promoción Científica y Tecnológica (ANPCyT) and the Consejo de Investigaciones de la Universidad Nacional de Tucumán (CIUNT) for their financial support. Dr van Sinderen is a member of the Alimentary Pharmabiotic Centre, which is a research centre funded by Science Foundation Ireland (SFI; Grant no.s 02/CE/B124 and 07/CE/B1368), through the Irish Government's National Development Plan.

\section{References}

Alm, L. (1982) Effect of fermentation on B-vitamin content of milk in Sweden. J Dairy Sci 65, 353-359.

Asrar, F.M. and O'Connor, D.L. (2005) Bacterially synthesized folate and supplemental folic acid are absorbed across the large intestine of piglets. J Nutr Biochem 16, 587-593. 
Bacher, A., Eberhardt, S. and Richter, G. (1996) Biosynthesis of riboflavin. In Escherichia coli and Salmonella: Cellular and Molecular Biology ed. Neidhardt, F.C., Curtiss, R. III, Ingraham, J.L.L., Lin, E.C.C., Low, K.B., Magasanik, B., Reznikoff, W.S., Riley, M., Schaechter, M. and Umbarger, H.E. pp. 657-664. Washington, DC, USA: ASM Press.

Bacher, A., Eberhardt, S., Fischer, M., Kis, K. and Richter, G. (2000) Biosynthesis of vitamin $b_{2}$ (riboflavin). Annu Rev Nutr 20, 153-167.

Bates, C.J. (1993) Folic acid. In Encyclopaedia of Food Science Food Technology and Nutrition ed. Macrae, R., Robinson, R.K. and Sadler, M.J. pp. 1936-1944. London, UK: Academic Press.

Battersby, A.R. (1994) How nature builds the pigments of life: the conquest of vitamin B. Science 264, 1551-1557.

Beck, W.S. (2001) Cobalamin (Vitamin $\mathrm{B}_{12}$ ). In Handbook of Vitamins, 3rd edn ed Rucker, R., Sutie, J., McCormick, D.B. and Machlin, L.J. pp. 463-512. New York: Marcell Dekker.

Blanck, H.M., Bowman, B.A., Serdula, M.K., Khan, L.K., Kohn, W. and Woodruff, B.A. (2002) Angular stomatitis and riboflavin status among adolescent Bhutanese refugees living in southeastern Nepal. Am J Clin Nutr 76, 430-435.

Blencowe, H., Cousens, S., Modell, B. and Lawn, J. (2010) Folic acid to reduce neonatal mortality from neural tube disorders. Int J Epidemiol 39(Suppl 1), i110-i121.

Burgess, C., O’Connell-Motherway, M., Sybesma, W., Hugenholtz, J. and van Sinderen, D. (2004) Riboflavin production in Lactococcus lactis: potential for in situ production of vitamin-enriched foods. Appl Environ Microbiol 70, 5769-5777.

Burgess, C.M., Smid, E.J., Rutten, G. and van Sinderen, D. (2006) A general method for selection of riboflavin-overproducing food grade micro-organisms. Microb Cell Fact 5, 24.

Burgess, C.M., Smid, E.J. and van Sinderen, D. (2009) Bacterial vitamin $\mathrm{B}_{2}, \mathrm{~B}_{11}$ and $\mathrm{B}_{12}$ overproduction: an overview. Int J Food Microbiol 133, 1-7.

Capozzi, V., Menga, V., Digesu, A.M., De Vita, P., van Sinderen, D., Cattivelli, L., Fares, C. and Spano, G. (2011) Biotechnological production of vitamin $\mathrm{B}_{2}$-enriched bread and pasta. J Agric Food Chem 59, 8013-8020.

Carroll, C., Cooper, K., Papaioannou, D., Hind, D., Tappenden, P., Pilgrim, H. and Booth, A. (2010) Meta-analysis: folic acid in the chemoprevention of colorectal adenomas and colorectal cancer. Aliment Pharmacol Ther 31, 708718.

Champagne, C.P., Tompkins, T.A., Buckley, N.D. and GreenJohnson, J.M. (2010) Effect of fermentation by pure and mixed cultures of Streptococcus thermophilus and Lactobacillus helveticus on isoflavone and B-vitamin content of a fermented soy beverage. Food Microbiol 27, 968-972.

Claesson, M.J., Li, Y., Leahy, S., Canchaya, C., van Pijkeren, J.P., Cerdeno-Tarraga, A.M., Parkhill, J., Flynn, S. et al. (2006) Multireplicon genome architecture of Lactobacillus salivarius. Proc Natl Acad Sci USA 103, 6718-6723.
Crittenden, R.G., Martinez, N.R. and Playne, M.J. (2003) Synthesis and utilisation of folate by yoghurt starter cultures and probiotic bacteria. Int J Food Microbiol 80, 217-222.

Deshpande, G., Rao, S. and Patole, S. (2011) Progress in the field of probiotics: year 2011. Curr Opin Gastroenterol 27, 13.

Elmadfa, I., Heinzle, C., Majchrzak, D. and Foissy, H. (2001) Influence of a probiotic yoghurt on the status of vitamins $\mathrm{B}(1), \mathrm{B}(2)$ and $\mathrm{B}(6)$ in the healthy adult human. Ann Nutr Metab 45, 13-18.

Encuesta Nacional de Nutrición y Salud (ENNyS) (2007) Buenos Aires, Argentina: Dirección Nacional de Salud Materno Infantil - Ministerio de Salud.

Escalante-Semerena, J.C. (2007) Conversion of cobinamide into adenosylcobamide in bacteria and archaea. J Bacteriol 189, 4555-4560.

Fabian, E., Majchrzak, D., Dieminger, B., Meyer, E. and Elmadfa, I. (2008) Influence of probiotic and conventional yoghurt on the status of vitamins $\mathrm{B}_{1}, \mathrm{~B}_{2}$ and $\mathrm{B}_{6}$ in young healthy women. Ann Nutr Metab 52, 29-36.

FAO/WHO (2001) Report of a Joint FAO/WHO expert consultation on evaluation of health and nutritional properties of probiotics in food including powder milk with live lactic acid bacteria.

Friend, B.A., Fiedler, J.M. and Shahani, K.M. (1983) Influence of culture selection on the flavor, antimicrobial activity, $\beta$ galactosidase and B-vitamins of yoghurt. Milchwissenschaft 38, 133-136.

Giovannucci, E. (2002) Epidemiologic studies of folate and colorectal neoplasia: a review. J Nutr 132, 2350S-2355S.

Glatzle, D., Korner, W.F., Christeller, S. and Wiss, O. (1970) Method for the detection of a biochemical riboflavin deficiency. Stimulation of NADPH2-dependent glutathione reductase from human erythrocytes by FAD in vitro. Investigations on the vitamin $\mathrm{B}_{2}$ status in healthly people and geriatric patients. Int $Z$ Vitaminforsch 40, 166-183.

van de Guchte, M., Penaud, S., Grimaldi, C., Barbe, V., Bryson, K., Nicolas, P., Robert, C., Oztas, S. et al. (2006) The complete genome sequence of Lactobacillus bulgaricus reveals extensive and ongoing reductive evolution. Proc Natl Acad Sci USA 103, 9274-9279.

Holasova, M., Fiedlerova, V., Roubal, P. and Pechacova, M. (2004) Biosynthesis of folates by lactic acid bacteria and propionibacteria in fermented milk. Czech J Food Sci 22, 175-181.

Hou, J.W., Yu, R.C. and Chou, C.C. (2000) Changes in some components of soymilk during fermentation with bifidobacteria. Food Res Int 33, 393-397.

Hufner, E., Britton, R.A., Roos, S., Jonsson, H. and Hertel, C. (2008) Global transcriptional response of Lactobacillus reuteri to the sourdough environment. Syst Appl Microbiol 31, 323-338.

Hugenholtz, J. and Smid, E.J. (2002) Nutraceutical production with food-grade microorganisms. Curr Opin Biotechnol 13, 497-507. 
Institute of Medicine ed. (1998) Dietary Reference Intakes for Thiamin, Riboflavin, Niacin, Vitamin $B_{6}$, Folate, Vitamin $B_{12}$, Panthothenic Acid, Biotin, and Choline. Washington, DC, USA: National Academies Press.

Jägerstad, M., Jastrebova, J. and Svensson, U. (2004) Folates in fermented vegetables - a pilot study. LWT - Food Sci Technol 37, 603-611.

Jayashree, S., Jayaraman, K. and Kalaichelvan, G. (2010) Isolation, screening and characterization of riboflavin producing lactic acid bacteria from Katpadi, Vellore district. Recent Res Sci Technol 2, 83-88.

Kariluoto, S., Aittamaa, M., Korhola, M., Salovaara, H., Vahteristo, L. and Piironen, V. (2006) Effects of yeasts and bacteria on the levels of folates in rye sourdoughs. Int $J$ Food Microbiol 106, 137-143.

Keck, B., Munder, M. and Renz, P. (1998) Biosynthesis of cobalamin in Salmonella typhimurium: transformation of riboflavin into the 5,6-dimethylbenzimidazole moiety. Arch Microbiol 171, 66-68.

Keuth, S. and Bisping, B. (1993) Formation of vitamins by pure cultures of tempe moulds and bacteria during the tempe solid substrate fermentation. J Appl Bacteriol 75, 427-434.

Kleerebezem, M., Boekhorst, J., van Kranenburg, R., Molenaar, D., Kuipers, O.P., Leer, R., Tarchini, R., Peters, S.A. et al. (2003) Complete genome sequence of Lactobacillus plantarum WCFS1. Proc Natl Acad Sci USA 100, 1990-1995.

Kneifel, W., Kaufmann, M., Fleischer, A. and Ulberth, F. (1992) Screening of commercially available mesophilic dairy starter cultures: biochemical, sensory, and microbiological properties. J Dairy Sci 75, 3158-3166.

Koizumi, S., Yonetani, Y., Maruyama, A. and Teshiba, S. (2000) Production of riboflavin by metabolically engineered Corynebacterium ammoniagenes. Appl Microbiol Biotechnol 53, 674-679.

Laiño, J., Savoy de Giori, G. and LeBlanc, J.G. (2011) Folate production by lactic acid bacteria. In Bioactive Foods and Supplements in Health Promotion - Liver and Gastrointestinal Disease ed. Watson, R. Oxford, UK: Elsevier.

Lamers, Y., Prinz-Langenohl, R., Bramswig, S. and Pietrzik, K. (2006) Red blood cell folate concentrations increase more after supplementation with [6S]-5-methyltetrahydrofolate than with folic acid in women of childbearing age. Am J Clin Nutr 84, 156-161.

LeBlanc, J.G., Burgess, C., Sesma, F., de Giori, G.S. and van Sinderen, D. (2005a) Lactococcus lactis is capable of improving the riboflavin status in deficient rats. $\mathrm{Br} J \mathrm{Nutr}$ 94, 262-267.

LeBlanc, J.G., Burgess, C., Sesma, F., Savoy de Giori, G. and van Sinderen, D. (2005b) Ingestion of milk fermented by genetically modified Lactococcus lactis improves the riboflavin status of deficient rats. J Dairy Sci 88, 3435-3442.

LeBlanc, J.G., Rutten, G., Bruinenberg, P., Sesma, F., de Giori, G.S. and Smid, E.J. (2006) A novel dairy product fermented with Propionibacterium freudenreichii improves the riboflavin status of deficient rats. Nutrition 22, 645-651.
LeBlanc, J.G., Savoy de Giori, G., Smid, E.J., Hugenholtz, J. and Sesma, F. (2007) Folate production by lactic acid bacteria and other food-grade microorganisms. In Communicating Current Research and Educational Topics and Trends in Applied Microbiology ed. Méndez-Vilas, A. pp. 329-339. Badajoz, Spain: Formatex Research Center.

LeBlanc, J.G., Sybesma, W., Starrenburg, M., Sesma, F., de Vos, W.M., de Giori, G.S. and Hugenholtz, J. (2010a) Supplementation with engineered Lactococcus lactis improves the folate status in deficient rats. Nutrition 26, 835-841.

LeBlanc, J.G., Taranto, M.P., Molina, V. and Sesma, F. (2010b) B-group vitamins production by probiotic lactic acid bacteria. In Biotechnology of Lactic Acid Bacteria: Novel Applications ed. Mozzi, F., Raya, R. and Vignolo, G. pp. 211-232. Ames, IA, USA: Wiley-Blackwell.

LeBlanc, J.G., van Sinderen, D., Hugenholtz, J., Piard, J.-C., Sesma, F. and Savoy de Giori, G. (2010c) Risk assessment of genetically modified lactic acid bacteria using the concept of substantial equivalence. Curr Microbiol 61, 590-595.

Lin, M.Y. and Young, C.M. (2000) Folate levels in cultures of lactic acid bacteria. Int Dairy J 10, 409-413.

Lucock, M. and Yates, Z. (2005) Folic acid - vitamin and panacea or genetic time bomb? Nat Rev Genet 6, 235-240.

Martens, J.H., Barg, H., Warren, M.J. and Jahn, D. (2002) Microbial production of vitamin $\mathrm{B}_{12}$. Appl Microbiol Biotechnol 58, 275-285.

Martin, R., Olivares, M., Marin, M.L., Xaus, J., Fernandez, L. and Rodriguez, J.M. (2005) Characterization of a reuterinproducing Lactobacillus coryniformis strain isolated from a goat's milk cheese. Int J Food Microbiol 104, 267-277.

Molina, V., Medici, M., Taranto, M.P. and Font de Valdez, G. (2008) Effects of maternal vitamin $B_{12}$ deficiency from end of gestation to weaning on the growth and haematological and immunological parameters in mouse dams and offspring. Arch Anim Nutr 62, 162-168.

Molina, V.C., Medici, M., Taranto, M.P. and Font de Valdez, G. (2009) Lactobacillus reuteri CRL 1098 prevents side effects produced by a nutritional vitamin B deficiency. J Appl Microbiol 106, 467-473.

Morishita, T., Tamura, N., Makino, T. and Kudo, S. (1999) Production of menaquinones by lactic acid bacteria. J Dairy Sci 82, 1897-1903.

Murdock, F.A. and Fields, M.L. (1984) B-vitamin content of natural lactic acid fermented cornmeal. J Food Sci 49, 373-375.

O’Brien, M.M., Kiely, M., Harrington, K.E., Robson, P.J., Strain, J.J. and Flynn, A. (2001) The North/South Ireland Food Consumption Survey: vitamin intakes in 18-64-yearold adults. Public Health Nutr 4, 1069-1079.

Ouwehand, A.C., Salminen, S. and Isolauri, E. (2002) Probiotics: an overview of beneficial effects. Antonie Van Leeuwenhoek 82, 279-289.

Papastoyiannidis, G., Polychroniadou, A., Michaelidou, A.M. and Alichanidis, E. (2006) Fermented milks fortified with B-group vitamins: vitamin stability and effect on resulting products. Food Sci Technol Int 12, 521-529. 
Perkins, J.B. and Pero, J. (2002) Vitamin biosynthesis. In Bacillus subtilis and its Closest Relatives from Genes to Cells eds Sonenshein, A., Hoch, J. and Losick, R. pp. 271-286. Washington, DC, USA: ASM Press.

Perkins, J.B., Sloma, A., Hermann, T., Theriault, K., Zachgo, E., Erdenberger, T., Hannett, N., Chatterjee, N.P. et al. (1999) Genetic engineering of Bacillus subtilis for the commercial production of riboflavin. J Ind Microbiol Biotech 22, 8-18.

Piao, Y., Kiatpapan, P., Yamashita, M. and Murooka, Y. (2004a) Effects of expression of hemA and hemB genes on production of porphyrin in Propionibacterium freudenreichii. Appl Environ Microbiol 70, 7561-7566.

Piao, Y., Yamashita, M., Kawaraichi, N., Asegawa, R., Ono, H. and Murooka, Y. (2004b) Production of vitamin $B_{12}$ in genetically engineered Propionibacterium freudenreichii. J Biosci Bioeng 98, 167-173.

Pompei, A., Cordisco, L., Amaretti, A., Zanoni, S., Matteuzzi, D. and Rossi, M. (2007) Folate production by bifidobacteria as a potential probiotic property. Appl Environ Microbiol 73, 179-185.

Putman, J. and Allshouse, J. (2003) Trends in U.S. Per Capita Consumption of Dairy Products, 1909 to 2001. Washington, DC: United States Department of Agriculture Economic Research Service.

Raux, E., Schubert, H.L. and Warren, M.J. (2000) Biosynthesis of cobalamin (vitamin $\mathrm{B}_{12}$ ): a bacterial conundrum. Cell Mol Life Sci 57, 1880-1893.

Rodionov, D.A., Vitreschak, A.G., Mironov, A.A. and Gelfand, M.S. (2003) Comparative genomics of the vitamin $B_{12}$ metabolism and regulation in prokaryotes. $\mathrm{J}$ Biol Chem 278, 41148-41159.

Roessner, C.A. and Scott, A.I. (2006) Fine-tuning our knowledge of the anaerobic route to cobalamin (vitamin $\mathrm{B}_{12}$ ). J Bacteriol 188, 7331-7334.

Rossi, M., Amaretti, A. and Raimondi, S. (2011) Folate production by probiotic bacteria. Nutrients 3, 118-134.

Roth, J.R., Lawrence, J.G. and Bobik, T.A. (1996) Cobalamin (coenzyme $\mathrm{B}_{12}$ ): synthesis and biological significance. Annu Rev Microbiol 50, 137-181.

Rucker, R.B., Suttie, J.W., McCormick, D.B. and Machlin, L.T. (2001) Handbook of Vitmains. New York: Marcel Dekker Inc.

Santos, F., Vera, J.L., Lamosa, P., de Valdez, G.F., de Vos, W.M., Santos, H., Sesma, F. and Hugenholtz, J. (2007) Pseudovitamin $\mathrm{B}(12)$ is the corrinoid produced by Lactobacillus reuteri CRL1098 under anaerobic conditions. FEBS Lett 581, 4865-4870.

Santos, F., Vera, J.L., van der Heijden, R., Valdez, G., de Vos, W.M., Sesma, F. and Hugenholtz, J. (2008a) The complete coenzyme $\mathrm{B}_{12}$ biosynthesis gene cluster of Lactobacillus reuteri CRL1098. Microbiology 154, 81-93.

Santos, F., Wegkamp, A., de Vos, W.M., Smid, E.J. and Hugenholtz, J. (2008b) High-level folate production in fermented foods by the $\mathrm{B}_{12}$ producer Lactobacillus reuteri JCM1112. Appl Environ Microbiol 74, 3291-3294.
Scott, A.I. (2003) Discovering nature's diverse pathways to vitamin $\mathrm{B}_{12}$ : a 35-year odyssey. J Org Chem 68, 2529-2539.

Shahani, K.M. and Chandan, R.C. (1979) Nutritional and healthful aspects of cultured and culture-containing dairy foods. J Dairy Sci 62, 1685-1694.

Smith, A.G., Croft, M.T., Moulin, M. and Webb, M.E. (2007) Plants need their vitamins too. Curr Opin Plant Biol 10, 266-275.

Soccol, C.R., de Souza Vandenberghe, L.P., Spier, M.R., Medeiros, A.B.P., Yamaguishi, C.T., Lindner, J.D.D., Pandey, A. and Thomaz-Soccol, V. (2011) The potential of probiotics: a review. Food Technol Biotechnol 48, 413-434.

Sybesma, W., LeBlanc, J.G., Starrenburg, M., Sesma, F., Savoy, G., de Vos, W.M. and Hugenholtz, J. (2003a) Lactococcus lactis, engineered for folate production, as source for bioavailable folates. In Metabolic Engineering of Folate Production in Lactic Acid Bacteria ed. Sybesma, W. pp. 193-212. The Netherlands: Wageningen University.

Sybesma, W., Starrenburg, M., Kleerebezem, M., Mierau, I., de Vos, W.M. and Hugenholtz, J. (2003b) Increased production of folate by metabolic engineering of Lactococcus lactis. Appl Environ Microbiol 69, 3069-3076.

Sybesma, W., Starrenburg, M., Tijsseling, L., Hoefnagel, M.H. and Hugenholtz, J. (2003c) Effects of cultivation conditions on folate production by lactic acid bacteria. Appl Environ Microbiol 69, 4542-4548.

Sybesma, W., Burgess, C., Starrenburg, M., van Sinderen, D. and Hugenholtz, J. (2004) Multivitamin production in Lactococcus lactis using metabolic engineering. Metab Eng 6, 109-115.

Taranto, M.P., Vera, J.L., Hugenholtz, J., de Valdez, G.F. and Sesma, F. (2003) Lactobacillus reuteri CRL1098 produces cobalamin. J Bacteriol 185, 5643-5647.

Thibaut, D., Blanche, F., Cameron, B., Crouzet, J., Debussche, L., Remy, E. and Vuilhorgne, M. (1998) Vitamin $B_{12}$ biosynthesis in Pseudomonas denitrificans. In Vitamin $B_{12}$ and $B_{12}$-Proteins eds Kräutler, B., Arigoni, D. and Golding, B.T. pp. 63-79. Weinheim, Germany: Wiley/VCH.

Ulrich, C.M. (2008) Folate and cancer prevention - where to next? Counterpoint. Cancer Epidemiol Biomarkers Prev 17, 2226-2230.

Vannini, V., de Valdez, G., Taranto, M.P. and Sesma, F. (2008) Identification of new lactobacilli able to produce cobalamin (Vitamin $\mathrm{B}_{12}$ ). Biocell 32, 72 .

Warren, M.J. (2006) Finding the final pieces of the vitamin $B_{12}$ biosynthetic jigsaw. Proc Natl Acad Sci USA 103, 4799-4800.

Warren, M.J., Raux, E., Schubert, H.L. and Escalante-Semerena, J.C. (2002) The biosynthesis of adenosylcobalamin (vitamin $\mathrm{B}_{12}$ ). Nat Prod Rep 19, 390-412.

Wegkamp, A., Starrenburg, M., de Vos, W.M., Hugenholtz, J. and Sybesma, W. (2004) Transformation of folate-consuming Lactobacillus gasseri into a folate producer. Appl Environ Microbiol 70, 3146-3148.

Wegkamp, A., van Oorschot, W., de Vos, W.M. and Smid, E.J. (2007) Characterization of the role of para-aminobenzoic 
acid biosynthesis in folate production by Lactococcus lactis. Appl Environ Microbiol 73, 2673-2681.

Wegkamp, A., Mars, A.E., Faijes, M., Molenaar, D., de Vos, R.C., Klaus, S.M., Hanson, A.D., de Vos, W.M. et al. (2010) Physiological responses to folate overproduction in Lactobacillus plantarum WCFS1. Microb Cell Fact 9, 1-14.

Wilson, J.A. (1983) Disorders of vitamins: deficiency, excess and errors of metabolism. In Harrison's Principles of Internal Medicine eds Petersdorf, R.G. and Harrison, T.R. pp. 461-470. New York, NY, USA: McGraw-Hill Book Co.
Wouters, J.T.M., Ayad, E.H.E., Hugenholtz, J. and Smit, G. (2002) Microbes from raw milk for fermented dairy products. Int Dairy J 12, 91-109.

Wu, Q.L., Chen, T., Gan, Y., Chen, X. and Zhao, X.M. (2007) Optimization of riboflavin production by recombinant Bacillus subtilis RH44 using statistical designs. Appl Microbiol Biotechnol 76, 783-794.

Yunxia, D., Tao, C., Xun, C., Jingyu, W. and Xueming, Z. (2010) Enhanced riboflavin production by expressing heterologous riboflavin operon from B. cereus ATCC14579 in Bacillus subtilis. Chin J Chem Eng 18, 129-136. 